\section{Six months versus two years of oral anticoagulation after a first episode of unprovoked deep-vein thrombosis. The PADIS-DVT randomized clinical trial}

Francis Couturaud, ${ }^{1}$ Gilles Pernod, ${ }^{2}$ Emilie Presles, ${ }^{3}$ Elisabeth Duhamel, ${ }^{4}$ Patrick Jego, ${ }^{5}$ Karine Provost, ${ }^{6}$ Brigitte Pan-Petesch, ${ }^{7}$ Claire Bal dit Sollier, ${ }^{8}$ Cécile Tromeur, ${ }^{1}$ Clément Hoffmann, ${ }^{9}$ Luc Bressollette, ${ }^{9}$ Philippe Lorillon, ${ }^{10}$ Philippe Girard, ${ }^{11}$ Emmanuelle Le Moigne, ${ }^{1}$ Aurélia Le Hir, ${ }^{1}$ Marie Guégan, ${ }^{1}$ Silvy Laporte ${ }^{3}$ Patrick Mismetti, ${ }^{12}$ Karine Lacut, ${ }^{1}$ Jean-Luc Bosson, ${ }^{13}$ Laurent Bertoletti, ${ }^{12}$ Oliver Sanchez, ${ }^{14}$ Guy Meyer, ${ }^{14}$ Christophe Leroyer ${ }^{1}$ and Dominique Mottier $^{1}$ for the "PADIS-DVT" investigators. *

${ }^{1}$ Département de Médecine Interne et Pneumologie, CHU de Brest, Université de Bretagne Occidentale, EA 3878, CIC INSERM 1412, F-CRIN INNOVTE, Brest;

2Département de Médecine Vasculaire, CHU de Grenoble, Université de Grenoble 1, FCRIN INNOVTE, Grenoble; 'Unité de Recherche Clinique, Innovation et Pharmacologie, CHU de Saint-Etienne, and INSERM U1059 SAINBIOSE, Université Jean Monnet, F-CRIN INNOVTE, Saint-Etienne; ${ }^{4}$ Service de Médecine Interne, Centre Hospitalier Général de Saint-Brieuc, F-CRIN INNOVTE, Saint-Brieuc; ${ }^{5}$ Service de Médecine Interne, CHU de Rennes, Université de Rennes 1, Rennes; 'Service de Cardiologie, Centre Hospitalier Général de Lannion, Lannion; 'Service d'Hématologie, Centre Hospitalier Général de Quimper, Quimper; ${ }^{8} \mathrm{Clinique}$ des Anticoagulants d'lle de France (C.R.E.A.T.I.F.), CHU de Lariboisière, Paris; ' Service d'Echo-Doppler Vasculaire, and EA 3878, CIC INSERM 1412, $\mathrm{CHU}$ de Brest, Université de Bretagne Occidentale, F-CRIN INNOVTE, Brest; ${ }^{10}$ Pharmacie Centrale, CHU de Brest, Université de Bretagne Occidentale, Brest; ${ }^{11}$ Département Thoracique, Institut Mutualiste Montsouris, F-CRIN INNOVTE, Paris; ${ }^{12}$ Service de Médecine Vasculaire et Thérapeutique, Unité de Pharmacologie Clinique, CIC1408, CHU de SaintEtienne, and INSERM U1059 SAINBIOSE, Université Jean Monnet, F-CRIN INNOVTE, SaintEtienne and ${ }^{13} \mathrm{CIC}$ and UMR CNRS 5525, CHU de Grenoble, Université de Grenoble 1, Grenoble, France.

*Members of the Prolongation d'un traitement par antivitamine $K$ pendant dix-huit mois versus placebo au décours d'un premier épisode de thrombose veineuse profonde Idiopathique traité six mois (PADIS-DVT) Study Group are listed in the Online Appendix.

\section{ABSTRACT}

T The optimal duration of anticoagulation after a first episode of unprovoked deep-vein thrombosis is uncertain. We aimed to assess the benefits and risks of an additional 18 months of treatment with warfarin versus placebo, after an initial 6 months of anticoagulation for a first unprovoked proximal deep-vein thrombosis. We conducted a multicenter, randomized, double-blind, controlled trial comparing an additional 18 months of warfarin with placebo in patients with a unprovoked proximal deep-vein thrombosis initially treated for 6 months (treatment period: 18 months; follow up after treatment period: 24 months). The primary outcome was the composite of recurrent venous thromboembolism or major bleeding at 18 months. Secondary outcomes were the composite at 42 months, as well as each component of the composite, and death unrelated to pulmonary embolism or major bleeding, at 18 and 42 months. All outcomes were centrally adjudicated. A total of 104 patients, enrolled between July 2007 and October 2013 were analyzed on an intention-to-treat basis; no patient was lost to follow-up. During the 18-month treatment period, the primary outcome occurred in none of the 50 patients in the warfarin group and in 16 out of 54 patients (cumulative risk, 29.6\%) in the placebo group (hazard ratio, 0.03 ; 95\% confidence interval: 0.01 to $0.09 ; P<0.001$ ). During the entire 42 -month study period, the composite outcome occurred
Ferrata Storti Foundation

Haematologica 2019

Volume 104(7):1493-1501

These results were presented on July 9 , 2017 , in the abstract session of the $56^{\text {th }}$ International Society of Thrombosis and Haemostasis Annual Meeting, Berlin, CA, July 6-9, 2017.

\section{Correspondence:}

FRANCIS COUTURAUD

francis.couturaud@chu-brest.fr

Received: November 5, 2018.

Accepted: January 2, 2019.

Pre-published: January 3, 2019.

doi:10.3324/haematol.2018.210971

Check the online version for the most updated information on this article, online supplements, and information on authorship \& disclosures: www.haematologica.org/content/104/7/1493

(C)2019 Ferrata Storti Foundation

Material published in Haematologica is covered by copyright. All rights are reserved to the Ferrata Storti Foundation. Use of published material is allowed under the following terms and conditions:

https://creativecommons.org/licenses/by-nc/4.0/legalcode. Copies of published material are allowed for personal or internal use. Sharing published material for non-commercial purposes is subject to the following conditions:

https://creativecommons. org//icenses/by-nc/4.0/legalcode, sect. 3. Reproducing and sharing published material for commercial purposes is not allowed without permission in writing from the publisher. 
in 14 patients (cumulative risk, $36.8 \%$ ) in the warfarin group and 17 patients (cumulative risk, $31.5 \%$ ) in the placebo group (hazard ratio, $0.72 ; 95 \%$ confidence interval: 0.35-1.46). In conclusion, after a first unprovoked proximal deep-vein thrombosis initially treated for 6 months, an additional 18 months of warfarin therapy reduced the composite of recurrent venous thrombosis and major bleeding compared to placebo. However, this benefit was not maintained after stopping anticoagulation. Clinical registration: this trial was registered at Ww w. clinicaltrials.gov as \#NCT00740493.

\section{Introduction}

Patients with unprovoked venous thromboembolism have a high risk of recurrence after short-term oral anticoagulant therapy for 3 to 6 months. ${ }^{1-7}$ Current guidelines suggest indefinite anticoagulation in these patients, although this is supported by only a moderate level of evidence. $^{8}$

Extending anticoagulation beyond 6 months of therapy is highly effective in preventing recurrent venous thromboembolism as long as treatment is maintained, but exposes patients to an increased risk of bleeding proportionate to treatment duration. ${ }^{1-7,9-14}$

Only a few randomized trials have followed patients during a substantial period (up to 1 or 2 years) after stopping anticoagulation to determine whether or not the benefit of extended anticoagulant therapy is maintained..$^{5-7,15}$ In a recent randomized trial comparing an additional 18 months of warfarin with placebo after a first episode of unprovoked pulmonary embolism initially treated for 6 months, we confirmed that the benefit of extended therapy was not maintained during a 2 -year follow-up period after discontinuing anticoagulation. ${ }^{7}$ However, although the rate of recurrent venous thromboembolism after an unprovoked pulmonary embolism has been reported to be close to that observed after an unprovoked deep-vein thrombosis, there is evidence that the case-fatality rate of recurrence is about 4 -fold higher after pulmonary embolism than after deep-vein thrombosis. ${ }^{16,17}$ Consequently, findings concerning unprovoked pulmonary embolism might not apply to patients with unprovoked deep-vein thrombosis. ${ }^{14}$

We therefore conducted a multicenter, randomized, double-blind trial in patients with a first episode of unprovoked proximal deep-vein thrombosis given an initial 6month course of oral anticoagulant therapy, to assess the benefits and risks of an additional 18 months of treatment with warfarin versus placebo during the study treatment period and during the 24 months following treatment discontinuation. A further aim was to compare the therapeutic effect of an additional 18 months of treatment in patients presenting unprovoked deep-vein thrombosis with that observed in the PADIS-PE trial.

\section{Methods}

\section{Ethical review and study organization}

The Prolonged Anticoagulation During Eighteen Months versus Placebo After Initial Six-Month Treatment for a First Episode of Idiopathic Deep-Vein Thrombosis (PADIS-DVT) study was conducted in accordance with the ethical principles stated in the Declaration of Helsinki, Good Clinical Practice, and relevant French regulations regarding ethics and data protection. The protocol and all amendments were approved by a central independ- ent Ethics Committee and written informed consent was obtained from all patients before randomization. The study was supervised by an academic steering committee. Study outcomes (recurrence, bleeding and death) were periodically reviewed by an independent data and safety monitoring committee. The role of this committee was to monitor the progress of the trial and to recommend study discontinuation to the steering committee if it considered that patients' safety was compromised. The University Hospital of Brest, the study sponsor, had no role in the design or conduct of the trial, data analysis, or preparation of the manuscript.

\section{Participants}

Patients aged 18 years or older who had experienced a first episode of symptomatic unprovoked proximal deep-vein thrombosis and had been initially treated without interruption for 6 (range, 5.5 to 7) months with a vitamin $\mathrm{K}$ antagonist [target International Normalized Ratio (INR), 2.0 to 3.0] were eligible for inclusion. Unprovoked proximal deep-vein thrombosis was defined as objectively confirmed symptomatic deep-vein thrombosis located in the popliteal vein or above ${ }^{18}$ occurring in the absence of any major reversible risk factor for venous thromboembolism within 3 months before diagnosis and in the absence of active cancer or cancer resolved within less than 2 years prior to the diagnosis of the venous thromboembolism. Reversible risk factors included surgery with locoregional or general anesthesia lasting over $30 \mathrm{~min}$, trauma with or without lower limb immobilization in a plaster cast, and bed-rest for more than $72 \mathrm{~h} .{ }^{4,7,8}$ Estrogen-containing contraception (used by 6 women) was not considered as a major reversible risk factor. No deep-vein thrombosis was related to prolonged travel or hormone replacement therapy. All the index proximal deep-vein thromboses were objectively diagnosed using leg vein ultrasound. ${ }^{18}$

The main exclusion criteria were: previous confirmed pulmonary embolism or proximal deep-vein thrombosis, concomitant symptomatic pulmonary embolism, recurrent venous thromboembolism or bleeding during the initial 6 months of anticoagulation, known major thrombophilia, indication for vitamin $\mathrm{K}$ antagonist therapy for reasons other than venous thromboembolism, increased bleeding risk, and life expectancy $<18$ months (see the Online Supplement for the full list).

All participants were ambulatory patients enrolled in eight French hospital centers from July 13 $3^{\text {th }}, 2007$ to October $16^{\text {th }}, 2013$ (last patient/last visit: December 31 ${ }^{\text {st }}$, 2016).

\section{Randomization, masking and interventions}

Except for the study population, the PADIS-DVT trial design was similar to the previously published PADIS-PE trial. ${ }^{7}$ After the initial 6 months of anticoagulation, patients were included and randomized to receive either warfarin (target INR, 2.0 to 3.0) or placebo (target sham INR, 2.0 to 3.0) for an additional 18 months using a central computerized internet-based system. Based on a computer algorithm, the randomization list was generated by an independent statistician (ClinInfo SA, Lyon, France) in randomly permuted blocks of four or six, with stratification by center. This list was forwarded to a central anticoagulation clinic not involved 
in the patients' care, before enrollment of the first patient.

Patients were provided with numbered supplies of study drug containing either warfarin tablets of $2 \mathrm{mg}$ and $5 \mathrm{mg}$ or visually identical placebo tablets (packaged at the sponsor's central pharmacy). On day 0 (date of randomization), all patients underwent laboratory testing (including D-dimer measurement) and leg ultrasound examination. Screening for thrombophilia was performed at the time of statistical analysis from centralized frozen blood samples taken on day 0 .

After written informed consent had been obtained, all study INR values were determined at each patient's usual local laboratory and were sent directly to the central anticoagulation clinic, with patients and investigators remaining unaware of the local results in order to maintain the double-blind condition. For patients assigned to warfarin, the clinic returned the true INR results to investigators for dose adjustments. For those assigned to placebo, the clinic substituted computer-generated sham INR results. The frequency of INR monitoring was left to the investigators' discretion, but was mandatory at least monthly and after each change in dose or concomitant therapy.

After the end of the treatment period, all patients were followed for an additional period of 24 months without anticoagulant therapy. Visits were scheduled at 3, 6, 12, 18, 30 and 42 months, and phone call contacts at 24 and 36 months.

\section{Outcome measures}

The primary outcome was the composite of symptomatic recurrent venous thromboembolism (including objectively confirmed non-fatal symptomatic pulmonary embolism or proximal deep-vein thrombosis or fatal venous thromboembolism) and non-fatal or fatal major bleeding, up to 18 months after inclusion. This composite outcome and its components were also assessed during the entire study period (i.e. up to 42 months). Other secondary outcomes were death unrelated to pulmonary embolism or major bleeding during the 18-month treatment period and the entire 42 -month study period. Secondary outcomes were specified in the statistical analysis plan (see Online Supplement) after the protocol was finalized, but before the database was locked and any data were analyzed.

Symptomatic recurrent deep-vein thrombosis or pulmonary embolism was objectively confirmed by ultrasonography, ventilation/perfusion lung scanning, spiral computerized tomography angiography, or autopsy, or in the event of sudden death for which no cause other than pulmonary embolism could be identified. ${ }^{18-20}$ Bleeding was considered as major according to the International Society of Thrombosis and Haemostasis definition (see Online Supplement). ${ }^{21}$ The American College of Chest Physicians (ACCP) bleeding score, initially unplanned, was calculated at the time of statistical analyses using prospectively recorded variables. ${ }^{8}$ All outcomes were adjudicated blindly by an independent central critical events committee. This committee had full access to any relevant medical reports and images from objective tests to adjudicate suspected events notified by investigators or detected during routine site monitoring.

\section{Sample size and statistical methods}

The trial was designed to demonstrate superiority of warfarin over placebo in preventing the composite primary outcome during the 18-month study treatment. Based on previous studies, ${ }^{1-7}$ with placebo we assumed rates of $9 \%$ per year of recurrent venous thromboembolism and $1 \%$ per year of major bleeding, yielding a $15 \%$ rate of the composite outcome at 18 months; corresponding assumptions with warfarin were $1 \%$ per year and $3 \%$ per year, yielding a $6 \%$ rate of the composite outcome at 18 months. A sample size of 178 patients per group therefore had $80 \%$ power to detect a statistically significant difference between groups (at a two-sided 5\% level of significance). Taking into account an anticipated $5 \%$ rate of patients lost to follow-up, a total sample of 374 patients was required.

In October 2013, after inclusion of the first 104 patients, the steering committee decided to end enrollment of patients into the study, as recommended by the data and safety monitoring committee in view of the slow recruitment rate that made reaching the expected sample size unfeasible. It is important to note that this recommendation was not prompted by any efficacy or safety issue. The data and safety monitoring committee also recommended that all included patients be followed until the last visit at 42 months, as originally planned in the protocol. These decisions were renewed every year and confirmed until the last included patient attended the last pre-planned visit at 42 months of follow up.

All analyses were performed on the intention-to-treat population, i.e. all randomized patients. Time-to-event outcomes were estimated by the Kaplan-Meier method, and between-group comparisons were performed using the log-rank test. The hazard ratio (HR) and corresponding 95\% confidence interval (CI) were calculated. If any baseline characteristic was differently distributed between the two groups $(P<0.05$ by the Fisher exact test, or Student $t$-test where appropriate), the comparisons were adjusted on the variables concerned using a Cox proportional hazards regression model. Time within the therapeutic INR range was calculated using standard methods, ${ }^{22}$ with corrections for planned interruptions of study drug. Finally, we combined the results from the PADIS-PE ${ }^{7}$ and PADIS-DVT studies by performing a metaanalysis of the log hazard ratios for the treatment effect on outcome from each study weighted by their inverse variances. All tests were two-sided and a $P$-value of less than 0.05 was considered to be statistically significant. Statistical analyses were performed using SAS V9.4 software (SAS Institute, Cary, NC, USA).

\section{Results}

Between July 2007 and October 2013, 104 patients were enrolled, 50 being randomized to warfarin and 54 to placebo. Of these patients, $102(98.1 \%)$ attended the 18-month visit and $100(96.2 \%)$ the 42 -month visit; no patient was lost to follow up (Figure 1 and Online Supplementary Table S1). The median follow up (interquartile range) was 23.6 (23.2-24.1) months after the treatment period, and 41.2 (40.9-41.6) months overall. Baseline characteristics were similar between the two groups (Table 1).

\section{Treatment and evaluation of International Normalized Ratios}

The median study treatment duration was similar in the two groups (warfarin: 17.5 months; placebo: 17.2 months). Double-blind treatment was permanently discontinued in six warfarin-treated patients (none with recurrent venous thromboembolism or major bleeding, all 6 discontinuing for other reasons), and 21 placebo-treated patients (16 with confirmed recurrent venous thromboembolism, none experiencing major bleeding and 5 discontinuing for other reasons). Of the six patients on warfarin who discontinued the study treatment for reasons other than major bleeding, two continued to receive anticoagulant therapy; of the five patients on placebo who discontinued for other reasons, two started anticoagulant therapy. The median interval between INR measurements was 19.5 days and 20.5 days for warfarin- and placebo- 
treated patients, respectively. In the warfarin group, INR was within the therapeutic range $(2.0-3.0]$ for $70.3 \%$ of the time, being below and above this range for $19.1 \%$ and $10.6 \%$ of the time, respectively; corresponding figures for the sham INR in the placebo group were $75.9 \%, 14.6 \%$ and $9.6 \%$, respectively (Online Supplementary Table S2).

\section{Clinical outcomes during the study treatment period}

During the 18-month study treatment period, the primary outcome occurred in none of the 50 patients in the warfarin group and in 16 of 54 patients in the placebo group (cumulative risk, 29.6\%; 28.3 events per 100 person-years), resulting in a relative risk reduction of $97 \%$ in favor of warfarin (HR, 0.03; 95\% CI: 0.01-0.51; $P<0.001$ ) (Table 2). As no major bleeding occurred in either group, the primary outcome was driven solely by the occurrence of symptomatic recurrent venous thromboembolism. All 16 episodes of symptomatic recurrent venous thromboembolism reported in the placebo group were unprovoked: one was fatal pulmonary embolism, 13 were isolated deep-vein thrombosis (2 contralateral and 11 ipsilateral) and two were non-fatal pulmonary embolism (1 was

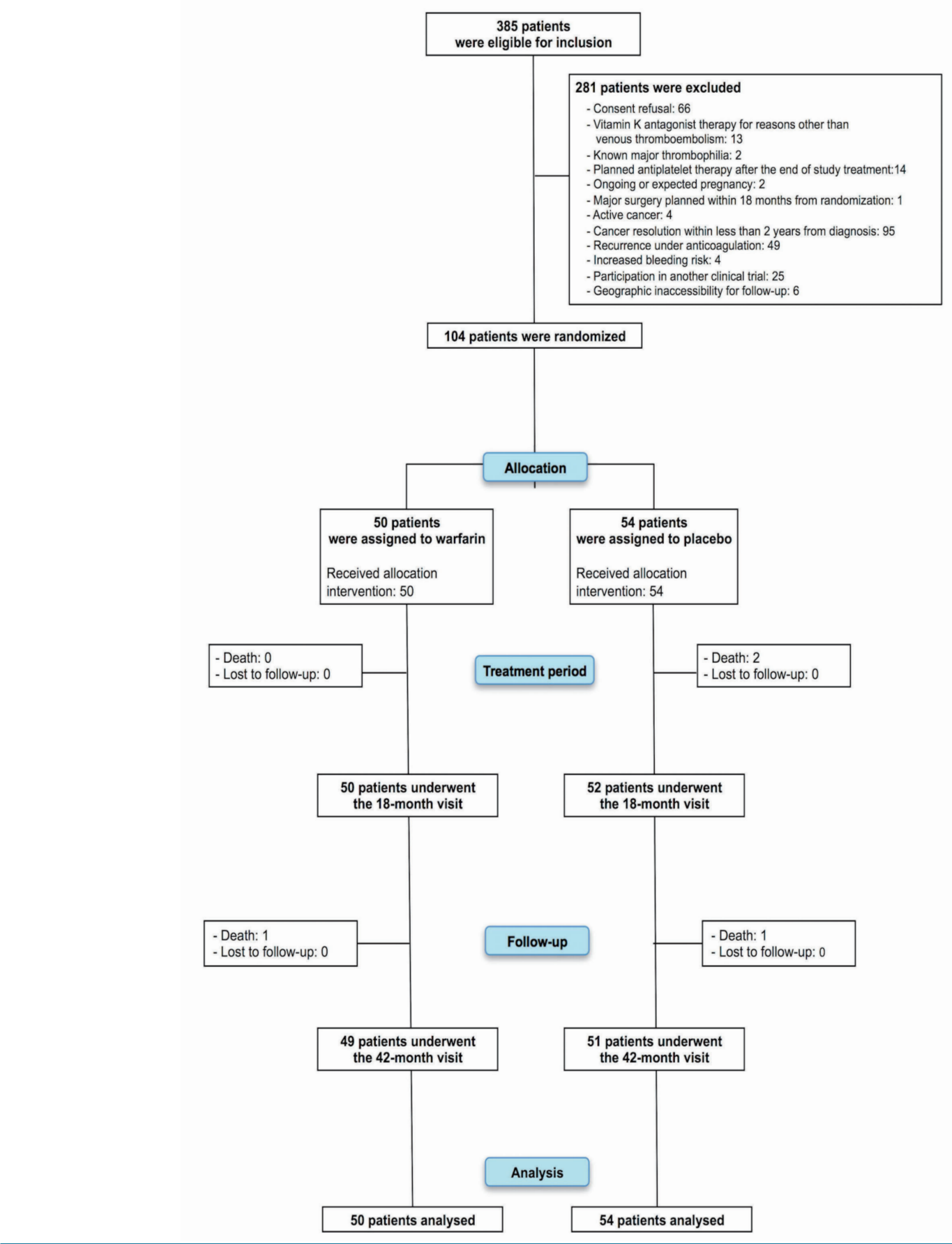

Figure 1. Patients' flow through the study. 
associated with an ipsilateral deep-vein thrombosis and 1 with a contralateral deep-vein thrombosis).

\section{Clinical outcomes after the study treatment period}

During the 24-month follow up after study treatment discontinuation, the composite outcome occurred in 14 patients in the warfarin group (cumulative risk, 26.3\%; 16.5 events per 100 person-years) and in one patient in the placebo group (cumulative risk, 2.0\%; 1.0 events per 100 person-years) (Table 2 ). In the warfarin group, symptomatic recurrent venous thromboembolism occurred in 14 patients, all in the absence of anticoagulation, and in 12 $(86.0 \%)$ cases was unprovoked. Of these 14 recurrences 13 were non-fatal isolated deep-vein thrombosis (6 contralateral and 7 ipsilateral), and one was non-fatal pulmonary embolism. In the placebo group, symptomatic recurrent venous thromboembolism occurred in one patient as an isolated ipsilateral deep-vein thrombosis, in the absence of anticoagulation, and was unprovoked and non-fatal. In the warfarin group, one patient had non-fatal major bleeding on warfarin.

\section{Clinical outcomes during the entire study period}

Overall, the composite outcome of recurrent venous thromboembolism or major bleeding occurred in 14 warfarin-treated patients (cumulative risk, 36.8\%; 11.2 events per 100 person-years) and 17 placebo-treated patients (cumulative risk, 31.5\%; 12.9 events per 100 personyears), resulting in a non-significant difference (HR, 0.72; 95\% CI: 0.35-1.46) (Table 2, Figure 2). Figure 2 shows the time course of the composite outcome. Results of prespecified subgroup analyses on the composite outcome during the treatment and entire study periods were consistent with the overall treatment effect (Online Supplementary Figures $S 1 A$ and $S 2 B)$.

\section{Meta-analysis}

The combined results of the PADIS-DVT and PADIS-PE ${ }^{7}$ trials showed a significant reduction of $87 \%$ in the rate of the composite outcome and of $93 \%$ in the rate of recurrent venous thromboembolism during the 18-month treatment period. A non-significant reduction of $27 \%$ in the rate of the composite outcome and of $32 \%$ in the rate of recurrent venous thromboembolism was observed over the 42-month study period (Figure 3).

\section{Discussion}

In this multicenter, randomized, double-blind trial in patients having completed 6 months of oral anticoagulation for a first episode of unprovoked proximal deep-vein thrombosis, an additional 18 months of warfarin therapy, as compared to placebo, was associated with a major $97 \%$ relative reduction in the risk of the composite endpoint of recurrent venous thromboembolism and major bleeding. However, this benefit was not maintained after anticoagulation was stopped.

In comparison with the PADIS-PE study, ${ }^{7}$ a similarly designed trial in patients with a first unprovoked pulmonary embolism, and other randomized studies having evaluated prolonged anticoagulation after unprovoked venous thromboembolism, ${ }^{4-6}$ we found an unexpectedly high risk of recurrent venous thromboembolism in the placebo group (cumulative risk of $37 \%$ at 18 months after
Table 1. Baseline characteristics of study participants according to randomized assignment. ${ }^{\mathrm{a}}$

\begin{tabular}{|c|c|c|}
\hline & $\begin{array}{l}\text { Warfarin } \\
(\mathrm{N}=50)\end{array}$ & $\begin{array}{l}\text { Placebo } \\
(\mathrm{N}=54)\end{array}$ \\
\hline $\begin{array}{l}\text { Age, mean }(\mathrm{SD}) \text {, years } \\
>65 \text { years, n. }(\%)\end{array}$ & $\begin{array}{l}59.0(17.2) \\
19(38.0)\end{array}$ & $\begin{array}{c}61.5(14.5) \\
29(53.7)\end{array}$ \\
\hline Female, n. (\%) & $19(38.0)$ & $15(27.8)$ \\
\hline $\begin{array}{l}\text { Body-mass index, mean (SD), } \mathrm{kg} / \mathrm{m}^{2} \\
\geq 30 \mathrm{~kg} / \mathrm{m}^{2} \text {, n. (\%) }\end{array}$ & $\begin{array}{l}26.8(4.9) \\
11(22.0)\end{array}$ & $\begin{array}{l}26.4(3.2) \\
6(11.1)\end{array}$ \\
\hline $\begin{array}{l}\text { Creatinine clearance category, }{ }^{\mathrm{b}} \text { no. (\%) } \\
<30 \mathrm{~mL} / \mathrm{min} \\
\geq 30 \text { and }<50 \mathrm{~mL} / \mathrm{min} \\
\geq 50 \mathrm{~mL} / \mathrm{min}\end{array}$ & $\begin{array}{c}1(4.8) \\
5(23.8) \\
15(71.4)\end{array}$ & $\begin{array}{c}0(0.0) \\
1(3.4) \\
28(96.6)\end{array}$ \\
\hline $\begin{array}{l}\text { Medical conditions, n. (\%) } \\
\text { Previous cancer }{ }^{c} \\
\text { Previous distal DVT } \\
\text { or superficial-vein thrombosis } \\
\text { Chronic heart failure } \\
\text { Chronic respiratory failure }\end{array}$ & $\begin{array}{l}2(4.0) \\
6(12.0) \\
3(6.0) \\
7(14.0)\end{array}$ & $\begin{array}{l}0(0.0) \\
9(16.7)\end{array}$ \\
\hline $\begin{array}{l}\text { Extent of DVT at diagnosis } \\
\text { Common femoral vein } \\
\text { Superficial femoral vein } \\
\text { Popliteal vein }\end{array}$ & $\begin{array}{l}19(40.4) \\
15(31.9) \\
13(27.7)\end{array}$ & $\begin{array}{l}15(27.8) \\
23(42.6) \\
16(29.6)\end{array}$ \\
\hline 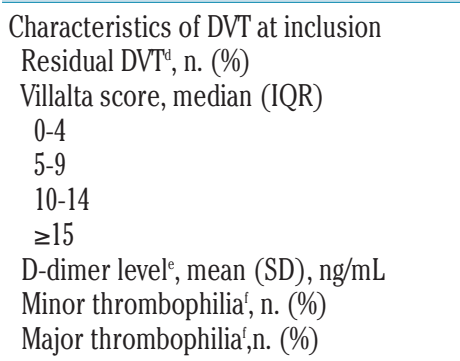 & $\begin{array}{c}27(55.1) \\
1.0(0.0-4.0) \\
43(86.0) \\
6(12.0) \\
1(2.0) \\
0 \\
327.4(315.3) \\
8(16.0) \\
9(18.0)\end{array}$ & $\begin{array}{c}32(59.3) \\
1.0(0.0-4.5) \\
39(75.0) \\
10(19.2) \\
2(3.8) \\
1(1.9) \\
287.0(227.5) \\
8(15.4) \\
14(26.9)\end{array}$ \\
\hline $\begin{array}{l}\text { Treatment of DVT prior to randomization } \\
\text { Warfarin, n. (\%) } \\
\text { Fluindione, n. (\%) } \\
\text { Acecoumarol, n. (\%) } \\
\text { Duration of initial anticoagulation, } \\
\text { mean (SD), months } \\
\text { Mean (SD) percentage time } \\
\text { in therapeutic INR range } \\
\text { Use of compression stockings, n. (\%) }\end{array}$ & $\begin{array}{l}25(50.0) \\
26(52.0) \\
0 \\
6.4(0.7) \\
68.7(26.4) \\
49(98.0)\end{array}$ & $\begin{array}{c}28(51.9) \\
26(48.1) \\
0 \\
6.3(0.5) \\
74.6(21.0)\end{array}$ \\
\hline $\begin{array}{l}\text { Main concomitant treatments, n. (\%) } \\
\text { Antiplatelet agent } \\
\text { Statins } \\
\text { Estrogen-containing contraception }\end{array}$ & $\begin{array}{l}2(4.0) \\
4(8.0) \\
4(8.0)\end{array}$ & $\begin{array}{l}5(9.3) \\
5(9.3) \\
2(3.7)\end{array}$ \\
\hline $\begin{array}{l}\text { ACCP bleeding risk at inclusion", n. (\%) } \\
\text { Low (no risk factor) } \\
\text { Moderate (1 risk factor) } \\
\text { High ( } \geq 2 \text { risk factors) }\end{array}$ & $\begin{array}{l}5(26.3) \\
5(26.3) \\
9(47.4)\end{array}$ & $\begin{array}{c}7(26.9) \\
5(19.2) \\
14(53.8)\end{array}$ \\
\hline
\end{tabular}

${ }^{a}$ Denominators may be lower than 50 and 54 due to missing data for some variables. Baseline characteristics of the two groups were compared using the Student t-test for quantitative variables and Fisher exact test for qualitative variables. None of the resulting $P$ values was $<0.05$ ${ }^{\mathrm{b}} \mathrm{Creatinine}$ clearance was estimated by the Cockcroft-Gault method; creatinine clearance values were available for only 21 patients in the warfarin group and 29 patients in the placebo

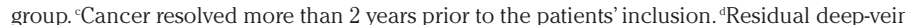
thrombosis, assessed using bilateral compression ultrasonography, was defined by the presence of persistent, non-full compressibility of a proximal deep-vein that was initially non-compressible in one or two lower limbs. ${ }^{~} \mathrm{D}$-dimer level was measured before randomization while patients were receiving vitamin $\mathrm{K}$ antagonist therapy. 'Screening for thrombophilia was per formed at the time of statistical analysis from centralized frozen blood samples taken on day 0 .Thrombophilia was defined as minor if patients had either heterozygous factorV Leiden or heterozygous G20210A prothrombin gene variant or elevated factor VIII (90th percentile) Thrombophilia was defined as major if patients had antithrombin or protein $\mathrm{C}$ or protein $\mathrm{S}$ deficiency or lupus anticoagulant or anticardiolipin antibodies $\left(99^{\text {th }}\right.$ percentile) or homozygous factor V Leiden or combined thrombophilia (details are provided in Online Supplementary Table S3). ${ }^{\text {STT }}$ The American College of Chest Physicians (ACCP) score was calculated at the time of the statistical analysis, using the patients' baseline characteristics. Definitions and references are provided in the Online Supplement. SD: standard deviation; DVT: deep-vein thrombosis; IQR: interquartile range; INR: International Normalized Ratio. 
Table 2. Primary and secondary outcomes according to study group assignment.

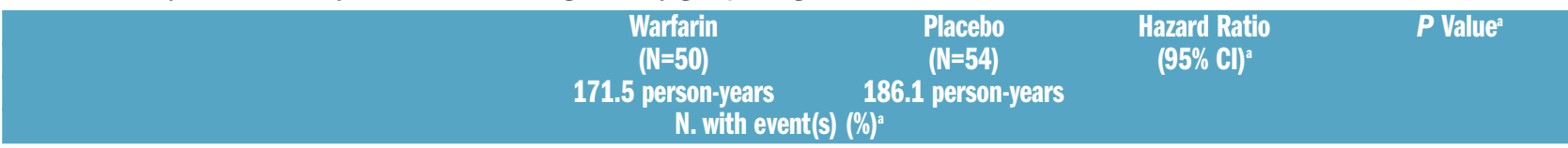

During the study treatment period of 18 months

Primary composite outcome, ${ }^{b}$ n. (\%)

$\begin{array}{cccc}0(0.0) & 16(29.6) & 0.03(0.01 \text { to } 0.51) & <0.001 \\ 0(0.0) & 16(29.6) & 0.03(0.01 \text { to } 0.51) & <0.001 \\ 0 & 1 & & \\ 0 & 2 & & \\ 0 & 12 & & \\ 0 & 1 \mathrm{~d} & - & 0.22 \\ 0 & 0 & & \\ 0 & 0 & & 0.33 \\ 0 & 0 & - & \\ 0(0.0) & 1(1.9) & & \end{array}$

Recurrent venous thromboembolism, n. (\%)

Fatal pulmonary embolism ${ }^{c}$

Symptomatic non-fatal pulmonary embolism

Symptomatic proximal deep-vein thrombosis

Symptomatic distal deep-vein thrombosis

Major bleeding, no. (\%)

Fatal

Non-fatal

Death from causes other than VTE

or major bleeding, n. (\%)

\section{During the entire study period of 42 months}

Composite outcome, n. (\%)

$14(36.8)$

Recurrent VTE, n. (\%)

Fatal pulmonary embolismc

Symptomatic non-fatal pulmonary embolism

Symptomatic proximal deep-vein thrombosis

Symptomatic distal deep-vein thrombosis

Major bleeding, n. (\%)

Fatal

Non-fatal

Death from causes other than VTE

or major bleeding, n. (\%)

$14(36.8)$

$(36.8) \mathrm{d}$
0
1
12
$1 \mathrm{~d}$

$1(2.0) \mathrm{e}$

${ }^{\mathrm{a}}$ Percentages are Kaplan-Meier estimates; all confidence intervals and $P$ values were estimated using a Cox proportional hazards regression model. ${ }^{\circ}$ The primary outcome was the composite of symptomatic recurrent venous thromboembolism (including objectively confirmed non-fatal symptomatic pulmonary embolism or proximal deep-vein thrombosis or fatal venous thromboembolism) and non-fatal or fatal major bleeding up to 18 months. Sudden death classified as fatal pulmonary embolism by the Critical Events Committee. ${ }^{\circledR}$ For two patients, symptomatic recurrent venous thrombosis occurred as contralateral distal deep-vein thrombosis; these two events were validated by the independent Critical Events Committee. 'One patient experienced recurrent venous thromboembolism 24 months after warfarin was discontinued owing to major bleeding. The same patient experienced rectorrhagia 10 months later (at 968 days), associated with a fall in hemoglobin level $\geq 2 \mathrm{~g} / \mathrm{dL}$, and requiring transfusion of two units of packed red cells HR: hazard ratio; $95 \%$ Cl: 95\% confidence interval;VTE: venous thromboembolism.

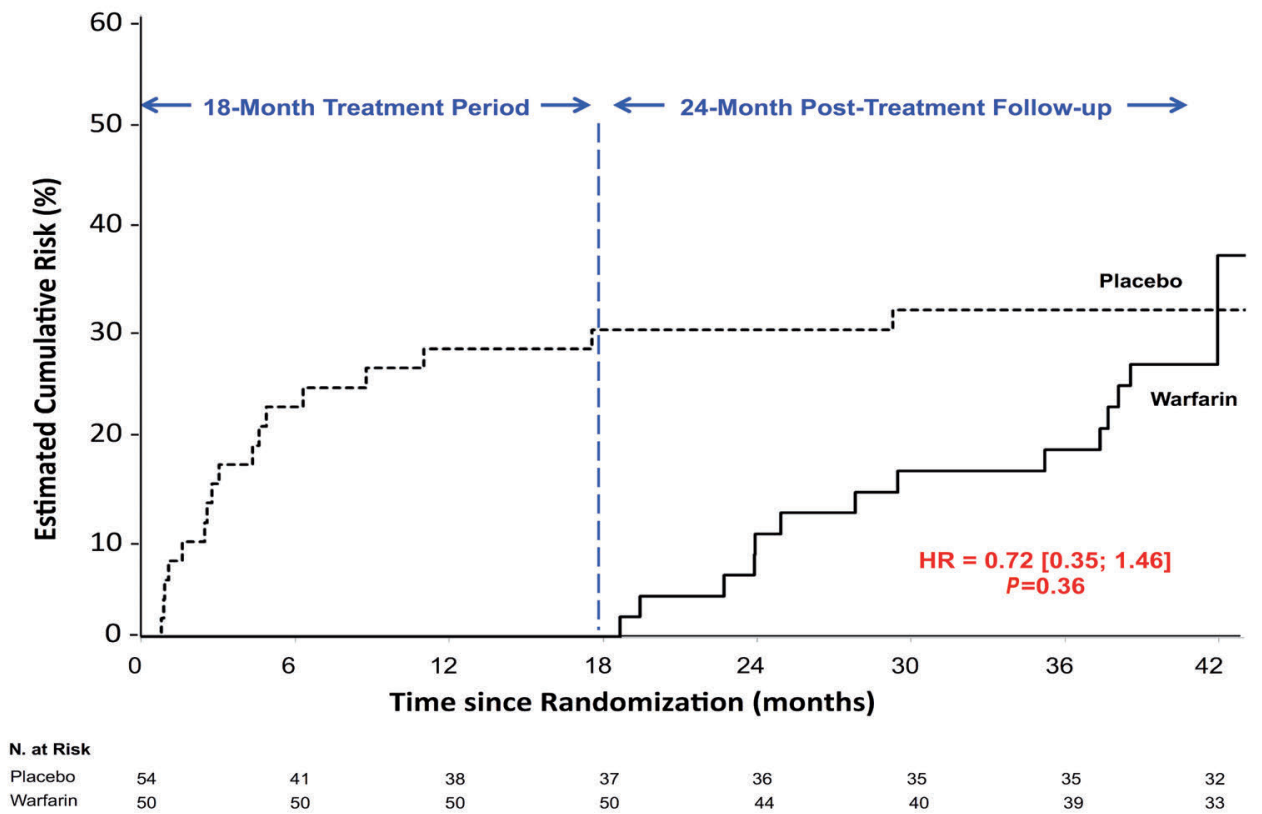

Figure 2. Kaplan-Meier estimates of the probability of the composite outcome of recurrent venous thromboembolism and major bleeding during the entire study period, according to study group. The unadjusted hazard ratios ( $95 \%$ confidence interval) warfarin/placebo were 0.03 (0.01 to 0.51 ) during the treatment period and 0.72 (0.35 to 1.46$)$ during the entire study period. 
inclusion). This observation is likely related to selection biases. First, at the time of study initiation, French national guidelines recommended 6 months of anticoagulation for a first unprovoked deep-vein thrombosis, making physicians reluctant to expose patients to an additional 18 months of treatment. ${ }^{23}$ Second, in France, relatively few patients with deep-vein thrombosis are currently referred to hospital centers, the vast majority being treated by community practitioners. Consequently, not only was recruitment much slower than expected but also mainly patients at high risk of recurrence were considered for inclusion, a factor consistent with the high prevalence of major thrombophilia in our study population (22\%). Although one could argue that such patients should have been excluded from the PADIS-DVT trial, systematic screening for thrombophilia was not a prerequisite for eli- gibility (see Online Supplement) and such a high prevalence of major thrombophilia is consistent with other studies on unprovoked VTE (e.g.; $8 \%$ and 15\% of antiphospholipid antibodies, homozygous factor $\mathrm{V}$ Leiden or homozygous G20218A prothrombin variant in the LAFIT and ELATE trials, respectively $)^{4,9}$ in which systematic detection of thrombophilia was not required. . $^{4,9,24}$ Nevertheless, extended warfarin therapy (for a target INR of $2.0-3.0$ ) dramatically reduced the risk of recurrent venous thromboembolism during the study treatment period to an extent similar to that observed in the PADIS-PE trial (Figure 3) and other trials on extended warfarin therapy. ${ }^{4-7}$ As previously reported, ${ }^{4-7}$ the risk of recurrence in the placebo group was greatest during the first months after stopping anticoagulation (cumulative risk, 38.7\%, 95\% CI: $19.1-58.3$ at 12 months) and then increased linearly by
At 18 months

\section{Primary composite outcome}

PADIS-PE $(N=371)$

PADIS-DVT $(\mathrm{N}=104)$

OVERALL

Heterogeneity: $I^{2}=70.6 \%, P=0.065$

Recurrent VTE

PADIS-PE $(N=371)$

PADIS-DVT $(\mathrm{N}=104)$

OVERALL

Heterogeneity: $I^{2}=16.1 \%, P=0.275$

\section{At 42 months}

Composite outcome

PADIS-PE $(\mathrm{N}=371)$

PADIS-DVT $(N=104)$

OVERALL

Heterogeneity: $I^{2}=0 \%, P=0.949$

Recurrent VTE

PADIS-PE $(N=371)$

PADIS-DVT $(\mathrm{N}=104)$

OVERALL

Heterogeneity: $\left.\right|^{2}=0 \%, P=0.870$
HR $[95 \% \mathrm{Cl}]$

$0.23[0.09 ; 0.55]$

$0.03[0.01 ; 0.51]$

$0.13[0.06 ; 0.32]$

$0.11[0.03 ; 0.37]$

$0.03[0.01 ; 0.51]$

$0.07[0.02 ; 0.22]$

$0.72[0.35 ; 1.46]$

$0.73[0.50 ; 1.08]$
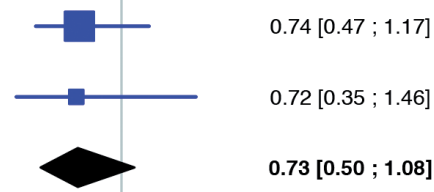

Figure 3. Meta-analysis of the PADIS-PE and PADIS-DVT studies. Pooled hazard ratios were calculated for the composite of recurrent venous thromboembolism and major bleeding and for the recurrent venous thromboembolism component. The pooled hazard ratio for the component of major bleeding is not reported as there was no major bleeding in the placebo group of the PADIS-DVT trial. HR hazard ratio; 95\% Cl: 95\% confidence interval; PE: pulmonary embolism; DVT: deep-vein thrombosis. 
$2-3 \%$ per year. The risk of recurrence in the warfarin group was lower than in the placebo group during the first months after anticoagulation discontinuation (cumulative risk, 9.4; 95\% CI: 2.87-15.9 at 12 months); however, if no rebound effect was observed as compared to the risk of recurrence in the placebo group in the first months following randomization, this risk increased linearly throughout the 24-month post-treatment period, resulting in an annual incidence of recurrence close to that observed in the placebo group during the entire study period. Finally, the loss of extended therapy benefit after cessation of anticoagulation corroborates that observed in other randomized trials evaluating extended therapy in patients with unprovoked venous thromboembolism. ${ }^{4-7}$

Finally, our results suggest that patients with a first episode of unprovoked proximal deep-vein thrombosis should receive indefinite anticoagulation; however, such an assumption is tempered by the following observation. Consistent with the results of the PADIS-PE and other trials, ${ }^{4-7,16}$ venous thromboembolism predominantly recurred in the same form as the index episode: regardless of whether patients had been treated for 6 or 24 months, $90 \%$ of recurrences were symptomatic deep-vein thrombosis, of which 3\% (1 of 31 episodes) were fatal, and $97 \%$ of all recurrences were unprovoked. Thus, most recurrences were certainly unpreventable but they represented the less severe form of venous thromboembolism.

Our study had several strengths: (i) we used a doubleblind, randomized design in a carefully predefined and characterized population; (ii) we achieved a very long and complete follow-up for all patients included; (iii) patients adhered closely to the study protocol during the treatment period and subsequent follow-up; and (iv) all outcomes were reviewed and validated blindly by an independent centralized adjudication committee.

The most important limitation is the small sample size of the study due to the premature discontinuation of study enrollment decided by the steering committee. This decision respected the safety committee's recommendations based on the slow recruitment rate, rendering attainment of the expected sample size unrealistic. However, all patients included had a complete follow up and our relative risk reduction estimates are consistent with those reported in our previous study in patients with unprovoked pulmonary embolism and other studies. ${ }^{46}$ Another limitation is that the primary outcome included two different outcome measures that may not be clinically equivalent, as the case-fatality rate of recurrent deep-vein thrombosis is inferior to that of major bleeding. ${ }^{15}$ However, the advantage of the composite endpoint is to provide a net benefit estimate encompassing the two main complications of venous thrombosis management (i.e., recurrent thrombosis and bleeding). In this setting, our results were essentially driven by a strong increase in the risk of recurrence that was unlikely to be offset by the risk of bleeding. Furthermore, a D-dimer-guided strategy was not used in our study, but this was not recommended when we designed the protocol and is still considered controversial. ${ }^{25,26}$ Lastly, extended therapy was evaluated using warfarin, not with direct oral anticoagulants. Our results suggest that patients such as ours require continued treatment. Whether direct oral anticoagulants, at full or low dose ${ }^{10-12}$ could constitute an effective and safe alternative to warfarin or could be tailored according to patients' risk factors (including elevated D-dimer levels), ${ }^{27}$ will need further investigation.

In conclusion, after a first episode of unprovoked proximal deep-vein thrombosis initially treated for 6 months, the major benefit of an additional 18 months of treatment with warfarin on the composite of recurrent venous thromboembolism and major bleeding was not maintained after discontinuation of anticoagulant therapy. Extended therapy did not modify the clinical presentation of recurrence, i.e., mostly unprovoked non-fatal proximal deep-vein thrombosis. Whether these findings apply to direct oral anticoagulants remains to be determined.

\section{Funding}

This study was supported by grants from the "Programme Hospitalier de Recherche Clinique" (French Department of Health) and the "Fondation de France". The study sponsor was the University Hospital of Brest. The study sponsor, had no role in the design or conduct of the trial, data analysis, or preparation of the manuscript.

\section{References}

1. Research Committee of the British Thoracic Society. Optimum duration of anticoagulation for deep-vein thrombosis and pulmonary embolism. Lancet. 1992;340(8824): 873-876.

2. Levine MN, Hirsh J, Gent M, et al. Optimal duration of oral anticoagulant therapy: a randomized trial comparing four weeks with three months of warfarin in patients with proximal deep-vein thrombosis. Thromb Haemost. 1995;74(2):606-611.

3. Schulman S, Rhedin A-S, Lindmarker P, et al. A comparison of six weeks with six months of oral anticoagulant therapy after a first episode of venous thromboembolism. Duration of Anticoagulation Trial Study Group. N Engl J Med. 1995;332(25):16611665.

4. Kearon C, Gent M, Hirsh J, et al. A comparison of three months of anticoagulation with extended anticoagulation for a first episode of idiopathic venous thromboembolism. $\mathrm{N}$ Engl J Med. 1999;340(12):901-907. Erratum in: N Engl J Med. 1999;341(4):298.

5. Agnelli G, Prandoni P, Santamaria MG, et al. Warfarin Optimal Duration Italian Trial Investigators. Three months versus one year of oral anticoagulant therapy for idiopathic deep venous thrombosis. Warfarin Optimal Duration Italian Trial Investigators. N Engl J Med. 2001;345(3):165-169.

6. Agnelli G, Prandoni P, Becattini C, et al. Warfarin Optimal Duration Italian Trial Investigators. Extended oral anticoagulant therapy after a first episode of pulmonary embolism. Ann Intern Med. 2003;139(1):1925.

7. Couturaud F, Sanchez O, Pernod G, et al. PADIS-PE investigators. six months vs extended oral anticoagulation after a first episode of pulmonary embolism: the PADIS-PE randomized clinical trial. JAMA. 2015;314(1):31-40.

8. Kearon C, Akl EA, Ornelas J, et al. Antithrombotic therapy for VTE disease:
CHEST guideline and expert panel report. Chest. 2016;149(2):315-352.

9. Kearon C, Ginsberg IS, Kovacs MJ, et al. Extended Low-Intensity Anticoagulation for Thrombo-Embolism Investigators. Comparison of low-intensity warfarin therapy with conventional-intensity warfarin therapy for long-term prevention of recurrent venous thromboembolism. N Engl J Med. 2003;349 (7):631-639

10. EINSTEIN Investigators, Bauersachs $\mathrm{R}$, Berkowitz SD, Brenner B, et al. Oral rivaroxaban for symptomatic venous thromboembolism. N Engl J Med. 2010;363(26):24992510.

11. Weitz JI, Lensing AWA, Prins MH, et al. EINSTEIN CHOICE Investigators. Rivaroxaban or aspirin for extended treatment of venous thromboembolism. N Engl J Med. 2017;376 (13):1211-1222

12. Agnelli G, Buller HR, Cohen A, et al AMPLIFY-EXT Investigators. Apixaban for extended treatment of venous thromboembolism. N Engl J Med. 2013;368(8):699-708. 
13. Schulman S, Kearon C, Kakkar AK, et al. REMEDY Trial Investigators; RE-SONATE Trial Investigators. Extended use of dabigatran, warfarin, or placebo in venous thromboembolism. N Engl J Med. 2013;368(8): 709-718.

14. Carrier M, Le Gal G, Wells PS, Rodger MA. Systematic review: case-fatality rates of recurrent venous thromboembolism and major bleeding events among patients treated for venous thromboembolism. Ann Intern Med. 2010;152(9):578-589.

15. Boutitie F, Pinede L, Schulman S, et al. Influence of preceding length of anticoagulant treatment and initial presentation of venous thromboembolism on risk of recurrence after stopping treatment: analysis of individual participants' data from seven trials. BMJ. 2011;342:d3036.

16. Douketis JD, Kearon C, Bates S, Duku EK, Ginsberg JS. Risk of fatal pulmonary embolism in patients with treated venous thromboembolism. JAMA. 1998;279(6):458462 .

17. Lecumberri R, Alfonso A, Jiménez D, et al. RIETE Investigators. Dynamics of casefatalilty rates of recurrent thromboem- bolism and major bleeding in patients treated for venous thromboembolism. Thromb Haemost. 2013;110(4):834-843

18. Kearon C, Julian JA, Newman TE, Ginsberg JS. Noninvasive diagnosis of deep venous thrombosis. McMaster Diagnostic Imaging Practice Guidelines Initiative. Ann Intern Med. 1998;128(8):663-677. Erratum in: Ann Intern Med. 1998;129(5):425.

19. Tapson VF. Acute pulmonary embolism. N Engl J Med. 2008;358(10):1037-1052.

20. Konstantinides S. Clinical practice. Acute pulmonary embolism. N Engl J Med. 2008;359(26):2804-2813.

21. Schulman S, Kearon C; Subcommittee on Control of Anticoagulation of the Scientific and Standardization Committee of the International Society on Thrombosis and Haemostasis. Definition of major bleeding in clinical investigations of antihemostatic medicinal products in non-surgical patients. J Thromb Haemost. 2005;3(4):692-694.

22. Rosendaal FR, Cannegieter SC, van der Meer FI, Briët E. A method to determine the optimal intensity of oral anticoagulant therapy. Thromb Haemost. 1993;69(3):236-239.

23. Mismetti P, Baud JM, Becker F, et al. Agence française de sécurité sanitaire des produits de santé. [Guidelines for good clinical practice: prevention and treatment of venous thromboembolism in medical patients]. J Mal Vasc. 2010;35(3):127-136.

24. Garcia D, Akl EA, Carr R, Kearon C. Antiphospholipid antibodies and the risk of recurrence after a first episode of venous thromboembolism: a systematic review. Blood 2013;122(5):817-824.

25. Baglin T, Bauer K, Douketis J, et al. Duration of anticoagulant therapy after a first episode of an unprovoked pulmonary embolus or deep-vein thrombosis: guidance from the SSC of the ISTH. J Thromb Haemost. 2012;10(4):698-702

26. Kearon C, Akl EA. Duration of anticoagulant therapy for deep-vein thrombosis and pulmonary embolism. Blood. 2014;123(12): 1794-801.

27. Rodger MA, Le Gal G, Anderson DR, et al REVERSE II Study Investigators. Validating the HERDOO2 rule to guide treatment duration for women with unprovoked venous thrombosis: multinational prospective cohort management study. BMJ. 2017;356:11065. 